\title{
Measurement of the
}

\section{Neutron Spectrum of a DD Electronic Neutron Generator}

\section{1st International Conference on the Application of Accelerators in Research and Industry}

The INL is a

U.S. Department of Energy

National Laboratory

operated by

Battelle Energy Alliance

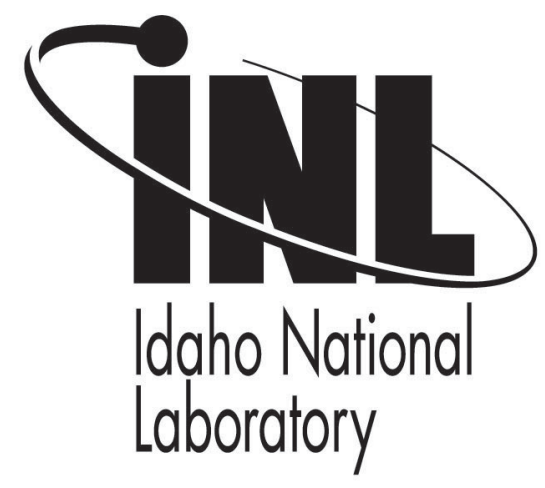

\section{David L. Chichester \\ James T. Johnson \\ Edward H. Seabury}

August 2010

This is a preprint of a paper intended for publication in a journal or proceedings. Since changes may be made before publication, this preprint should not be cited or reproduced without permission of the author. This document was prepared as an account of work sponsored by an agency of the United States Government. Neither the United States Government nor any agency thereof, or any of their employees, makes any warranty, expressed or implied, or assumes any legal liability or responsibility for any third party's use, or the results of such use, of any information, apparatus, product or process disclosed in this report, or represents that its use by such third party would not infringe privately owned rights. The views expressed in this paper are not necessarily those of the United States Government or the sponsoring agency. 


\title{
Measurement of the Neutron Spectrum of a DD Electronic Neutron Generator
}

\author{
David L. Chichester, James T. Johnson, and Edward H. Seabury \\ Idaho National Laboratory, 2525 N. Fremont Avenue, Idaho Falls, Idaho 83415
}

\begin{abstract}
A Cuttler-Shalev (C-S) ${ }^{3}$ He proportional counter has been used to measure the energy spectrum of neutrons from a portable deuterium-deuterium electronic neutron generator. To improve the analysis of results from the C-S detector digital pulse shape analysis techniques have been used to eliminate neutron recoil artifacts in the recorded data. Data was collected using a 8-GHz, 10-bit waveform digitizer with its full scale corresponding to approximately 6-MeV neutrons. The measurements were made with the detector axis perpendicular to the direction of ions in the ENG in a plane $0.5-\mathrm{m}$ to the side of the ENG, measuring neutrons emitted at an angle from $87.3^{\circ}$ to $92.7^{\circ}$ with respect to the path of ions in the ENG. The system demonstrated an energy resolution of approximately $0.040 \mathrm{MeV}$ for the thermal peak and approximately $0.13 \mathrm{MeV}$ at the DD neutron energy. In order to achieve the ultimate resolution capable with this type of detector it is clear that a higher-precision digitizer will be needed.
\end{abstract}

Keywords: Neutron spectrometry, digital pulse shape analysis, DD-fusion, electronic neutron generator.

PACS: $25.45 \mathrm{z}, 29.20 . \mathrm{Ej}, 29.25 . \mathrm{Dz}, 29.30 . \mathrm{Hs}, 84.30 . \mathrm{Sk}$

\section{INTRODUCTION}

Neutron spectrometry can provide a wealth of information about the structure of nuclei and the characteristics of nuclear reactions.[1] At a more applied level neutron spectrometry can provide primary and supplemental information as a nondestructive measurement technique to support industrial, medical, and security related activities. Unfortunately, it is difficult to accurately measure the energy of neutrons with both sufficient detection efficiency and sufficient energy-resolution to make meaningful and timely analyses in many cases. Time of flight measurements may be made using a nuclear reactor with a chopper wheel or with some neutronproducing particle accelerators; however, the infrastructure required for these measurements is often prohibitive for low turnaround or field applications. Detectors employing organic scintillators, both liquids and solids, with the ability to use pulse shape discrimination to separate neutron events from photon events have found some use for laboratory measurements. These detectors have reasonably sufficient detection efficiencies for many measurements but their energy resolution is typically limited to a few hundred keV.[2] The energy resolution achievable in these detectors is limited due to the need to employ sensitive mathematical unfolding algorithms to 'deconvolve' measured spectra, using simulated and/or measured response-function definitions to iteratively determine the spectrum of an incident neutron field.

An alternate detector approach for neutron spectrometry that has found some utility is the use of a ${ }^{3} \mathrm{He}$-ionization chamber coupled with pulse-height analysis, recording the energy of incident neutrons in a technique not unlike that used in scintillator-based photon spectrometry.[3-7] Detectors based on this approach are frequently referred to as Cuttler-Shalev (C-S) spectrometers. Neutron detection in this approach is a result of neutron absorption in the ionization chamber's ${ }^{3} \mathrm{He}$ fill gas in the ${ }^{3} \mathrm{He}(\mathrm{n}, \mathrm{p})^{3} \mathrm{H}$ reaction, the resulting proton and tritium atom proceed to ionize the ionization chamber's fill gas (which also often contains a denser gas such as methane, to reduce the path length of the charged particles) which then produce an electronic signal as these electrons and ions are collected.[8] Neutron capture in ${ }^{3} \mathrm{He}$ is exothermic with a Q-value of $0.764 \mathrm{MeV}$; the amplitude of signal pulses resulting from neutron capture corresponds to the sum of this energy plus the incident neutron's energy. These detectors are very sensitive to microphonic noise and electromagnetic interferences but under ideal conditions neutron energy measurements have been made with a resolution of $<15 \mathrm{keV}$, which is the limiting factor for the low-energy range of spectrometry for these 
detectors. The energy resolution of these detectors degrades at higher energies; a good study of the relationship between neutron energy and resolution for $\mathrm{C}-\mathrm{S}$ spectrometers is provided in the work of $\mathrm{Ohm}$ et al. and Owen et al. $[9,10]$ The C-S spectrometer energy resolution performance has been reported several times in the literature by several groups and includes observations of $E_{n}=$ thermal $(F W H M=0.013$ $\mathrm{MeV})$ [11], $\mathrm{E}_{\mathrm{n}}=$ thermal $(\mathrm{FWHM}=0.013 \mathrm{MeV})[12]$, $\mathrm{E}_{\mathrm{n}}=1 \mathrm{MeV}(\mathrm{FWHM}=0.025 \mathrm{MeV})[11], \mathrm{E}_{\mathrm{n}}=1 \mathrm{MeV}$ $(\mathrm{FWHM}=0.027 \mathrm{MeV})[10], \mathrm{E}_{\mathrm{n}}=1 \mathrm{MeV}(\mathrm{FWHM}=$ $0.029 \mathrm{MeV})$ [12], $\mathrm{E}_{\mathrm{n}}=1.27 \mathrm{MeV}(\mathrm{FWHM}=0.065$ $\mathrm{MeV})$ [4], and $\mathrm{E}_{\mathrm{n}}=2.450 \mathrm{MeV}(\mathrm{FWHM}=0.046$ $\mathrm{MeV})$ [13].

A key aspect of the use of a C-S spectrometer is the application of rise-time pulse shape discrimination to distinguish neutron capture events leading to fullenergy deposition in the detector from elastic scattering events and from wall-effect events, where one or both of the neutron capture reaction daughter products hits a wall before depositing all of its energy into the chamber gas.[3,12,14,15] Until recently most measurements systems used with C-S spectrometers used analog pulse analysis techniques to execute the rise-time pulse shape analyses. Continued development of laboratory electronics has also lead to the use of high-speed waveform digitizers has presented new options for collecting and analyzing this data. An early example of the application of the use of waveform digitization with a C-S spectrometer was presented by Takahashi et al. who used a 10-bit, 100$\mathrm{MHz}$ digitizer to record the thermal neutron capture peak in a C-S style neutron spectrometer and then analyzed their data using digital pulse shape analysis (dPSA) techniques.[16] This group did not perform rise-time analyses for artifact rejection and studied only the detection characteristics of their system for analyzing the thermal neutron peak.

In this paper we present research examining the use of dPSA techniques to analyze data from a C-S neutron spectrometer measuring neutrons from a deuterium-deuterium (DD) electronic neutron generator (ENG). A DD-ENG produces pseudomonoenergetic neutrons, with the actual energy of the neutron spectrum a) varying with respect to angle of the ENG's internal ion beam and b) having a distribution as a result of ion straggling within the ENGs metal hydride target. The kinematics of the reaction are well known and have been described in the literature.[17-19] The angular dependence of neutron yield versus angle is shown in FIGURE 1 together with a plot of neutron energy versus angle, for cases with $50-\mathrm{keV}$ and $100-\mathrm{keV}$ incident deuteron energies. Also shown in this figure is the impact a thick target has on the neutron energy. At the $90^{\circ}$ observation angle (perpendicular to the ENG) the neutron yield is relatively constant over a short range of $\pm 10^{\circ}$. Also, over this narrow range the relation between neutron energy $\left(2.46 \mathrm{MeV}\right.$ at $\left.90^{\circ}\right)$ and angle is nearly the same for incident deuteron energies from $0.050-\mathrm{MeV}$ to $0.100-\mathrm{MeV}$ and for thin and thick targets.
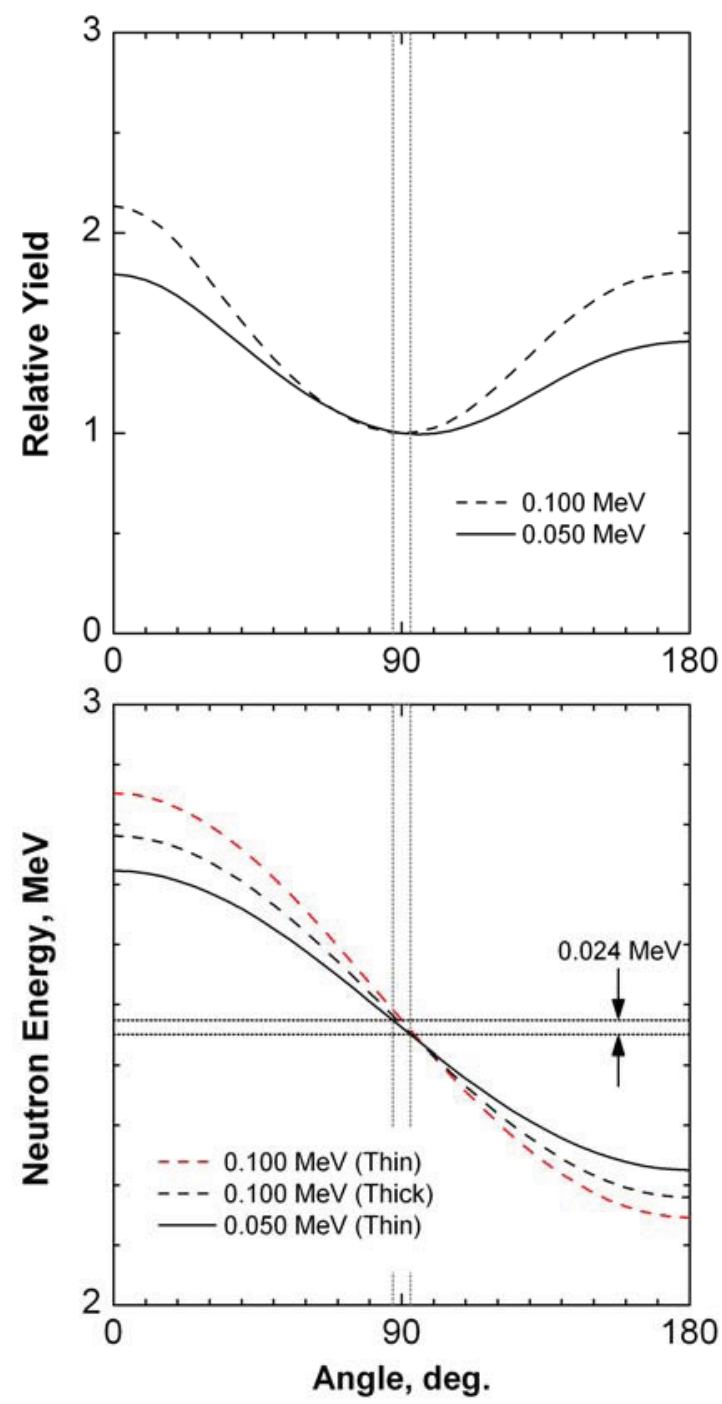

FIGURE 1. The upper figure shows the theoretical relative yield of neutrons as a function of angle for $0.050-\mathrm{MeV}$ and $0.100-\mathrm{MeV}$ deuterons striking a deuterated target inside an ideal ENG. The lower figure shows the theoretical energy of neutrons as a function of angle for $0.050-\mathrm{MeV}$ deuterons striking a thin target and for $0.100-\mathrm{MeV}$ deuterons striking both thin and thick targets. The vertical dotted lines represent the emitted neutron angle range detectable by the spectrometer used here. The horizontal dotted lines in the figure on the bottom represent the approximate spread in energy of neutrons incident on the C-S spectrometer as used in the measurement reported here.[17-19] 


\section{EXPERIMENTAL APPROACH}

Data was collected at Idaho National Laboratory in a shielded facility dedicated for testing and operating ENGs. [20] This facility has a $9.9 \mathrm{~m} \times 7.6 \mathrm{~m}$ test cell with a concrete floor and concrete walls; there is a relatively high degree of neutron scattering from the floor and walls that is easily observed using the C-S spectrometer. For the testing reported here the target plane of the ENG was in the center of the room, 1.48$\mathrm{m}$ above the floor. The ENG was oriented perpendicular to the floor with the internal deuteron beam pointed upwards. The spectrometer was placed 0.5-m away from the ENG, parallel to the floor with its broadside facing the ENG, supported by empty cardboard boxes so its centerline was $1.48-\mathrm{m}$ above the floor; neutrons from the ENG were incident on to the side of the detector. Data acquisition equipment was placed on a nearby table and then accessed remotely via computer in order to minimize variations to the scattering environment during the experiments.

\section{The Electronic Neutron Generator}

An MP320 compact electronic neutron generator loaded with deuterium from Thermo Electron was used for this project.[21] The ENG was not pulsed but rather was operated in a steady-state mode of operation. The accelerating voltage was $0.090 \mathrm{MeV}$, the average beam current was $60 \mu \mathrm{A}$. Previous measurements of this ENG have determined that these operating conditions produce a total neutron yield of $(2.36 \pm 0.65) \times 10^{6}$ neutrons per second. This ENG operates with a Penning-type ion source and is known to produce a mostly diatomic ion current of $\mathrm{D}_{2}^{+}$ions. A well-accepted rule of thumb with this device when operating at $0.090 \mathrm{MeV}$ is to approximate the device as having a $100 \%$ atomic beam current of $2 \mathrm{D}^{+}$ions at $0.050 \mathrm{MeV}$. For the data reported below the ENG was operated for one hour.

\section{The Detector}

The C-S neutron spectrometer used for this study was the FNS-100 Fast Neutron Spectrometer, a commercial instrument made by Bubble Technologies, Inc. (Ontario, Canada). The detector is functionally equivalent to the FNS-100 previously manufactured by Seforad-Applied Radiation Ltd. (Emek Hayaeden, Israel), a gridded ionization chamber that was used in much of the literature cited above. The detector has been extensively described in the literature including prior reports by Sailor and Prussin, Evans and Brandenberger, and Fisher et al.[6,12,13] The detector's sensitive length was $0.015 \mathrm{~m}$, its sensitive diameter was $0.0047 \mathrm{~m}$. An Ortec Model 456 power supply was used to bias the detector. The absolute efficiency for C-S type spectrometers, without pulse shape discrimination, has been reported to be $(1.7 \pm$ $0.5) \times 10^{-4}$ at $2.45 \mathrm{MeV} .[13]$

\section{The Data Acquisition System}

The output signal from the detector was recorded using an Acqiris DC282 10-bit high-speed waveform digitizer and stored on a high-capacity portable hard drive for post processing. This digitizer operates at 8 GHZ, collects data samples every 0.125 ns. The detector signals were scaled so that the digitizer would have a dynamic range capturing waveforms corresponding to full-energy neutron capture events up to approximately $6 \mathrm{MeV}$. Post processing was carried out on a computer using algorithms developed using National Instrument's LabView programming language. The algorithm used for this work was similar to the discrimination techniques implemented previously using analog components; analyzing the rise time of the digital pulses from the spectrometer and then discriminating longer rise-time events from shorter rise-time events. Improvements to this simple approach were developed including the application of filter to remove noise and a pulse shape analysis method to identify and eliminate pile-up events. Examples of some waveforms that passed the processing algorithm as 'good' events are shown in FIGURE 2; note that these events have been scaled to have approximately the same magnitude, demonstrating the significant differences in the shapes of these pulses.

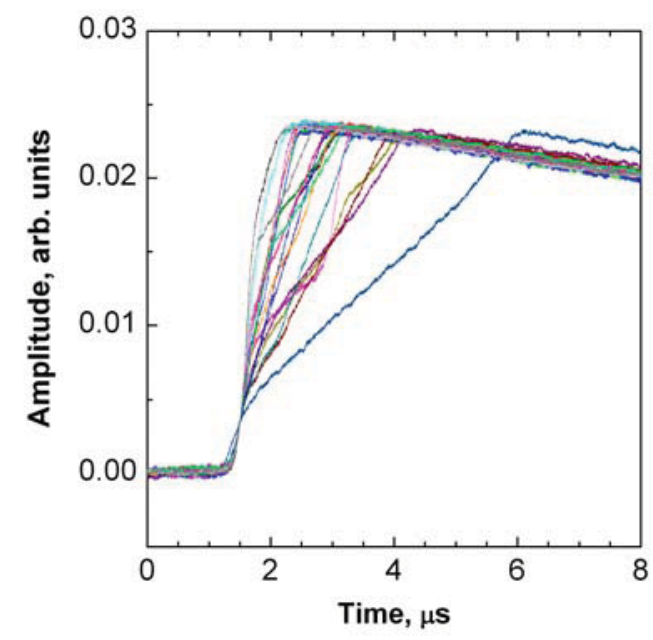

FIGURE 2. An example of digitized waveforms from the C-S spectrometer used in this paper (normalized). 


\section{RESULTS}

The raw neutron spectrum measured from the MP320 DD-ENG is shown in FIGURE 3. Also shown in this figure are the spectral components rejected using rise-time analysis as recoil events and using pulse shape analysis as pile-up events and wall-capture events. A closer look at the neutron capture peaks at $0.764 \mathrm{MeV}$ (thermal neutrons) and 3.22 MeV (DD neutrons) is given in FIGURE 4. A Gaussian fit to the thermal neutron capture peak yields an energy resolution of approximately $0.040 \mathrm{MeV}$ for the set-up used here. For the DD neutron capture peak a Gaussian fit having a full-width at half-maximum (FWHM) value of approximately $0.13 \mathrm{MeV}$ was calculated by deconvolving the observed peak shape with a top-hat function having a width of $0.024 \mathrm{MeV}$, the approximate spread in energy of neutrons incident on the detector determined by accounting for the detector-ENG subtended angle from $87.3^{\circ}$ to $92.7^{\circ}$. The energy calibration for the spectral data shown in these figures was determined with the processed spectrum and a two-point linear fit using the thermal neutron capture peak and the theoretical DD capture peak energy, taken to be $2.46 \mathrm{MeV}$. The uncertainty of this energy calibration is estimated to be approximately $0.01 \mathrm{MeV}$ at the DD energy.

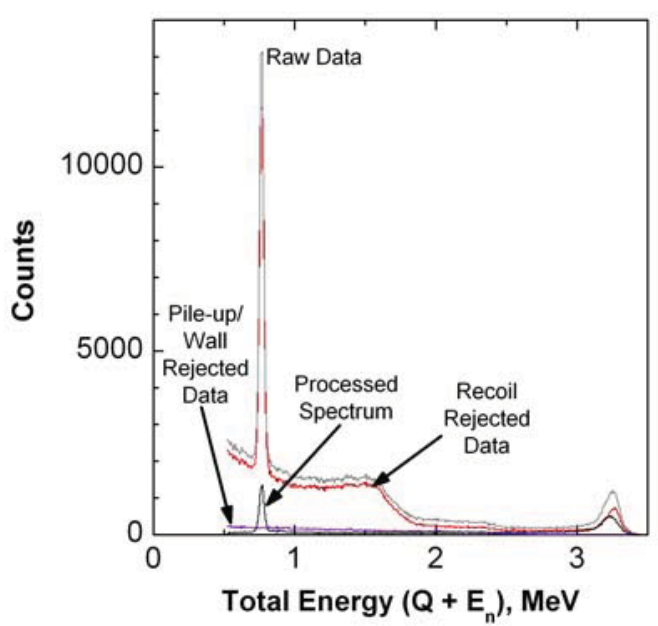

FIGURE 3. The raw pulse height spectrum acquired from the neutron spectrometer (grey) together with the recoil rejected data (red), pile-up/wall rejected data (purple), and the final processed and accepted spectrum (black).

\section{DISCUSSION}

The DD neutron spectrum observed using the C-S spectrometer as described here is in good qualitative agreement with previously reported measurements of monoenergetic neutron spectra in the DD energy- range. $[15,22,23]$ Of the total number of neutrons in the DD energy region approximately $57 \%$ were rejected by the recoil and pulse pile-up rejection algorithms, which is on par with prior work using analog electronics. The $0.040 \mathrm{MeV}$ energy resolution for the thermal peak observed here was significantly poorer than that previously achieved using analog electronics, or previously achieved by Takahashi et al. using digital electronics. This is likely due to the use of a 10-bit digitizer resolution applied over a roughly 6.8 MeV total energy range. Under these conditions the measurement system's theoretical best possible energy resolution was approximately $0.007 \mathrm{MeV}$. Accounting for noise effects in the digitized waveform, and our need to have a much wider energy range then that used by Takahashi et al., it seems clear that a 12-bit or possibly a 14-bit digitizer will be needed to achieve the best possible energy resolution achievable with the C-S spectrometer. We intend to perform tests with 12-bit and 14-bit digitizers the near future. The $8 \mathrm{GHz}$ sampling speed used for this data collection was more than what was needed. Examining FIGURE 2 it appears that a $100-\mathrm{MHz}$ instrument would have been adequate for this work.

An interesting feature of the processed spectrum is the low-energy tail observed on the DD neutron peak of FIGURE 4. Similar effects have been observed previously and reported in the literature. Takeda and Kudo have suggested this may be a real effect due to neutron scattering in the measurement environment.[15] Our prior work has investigated the important impact neutron scattering has on the neutron spectrum in the vicinity of compact neutron generators and, within the concrete room of the measurements described here, it would certainly have been a significant effect.[24] Another suggested cause for the low-energy tail are problems associated with the uniformity of gas-multiplication in the counter (Takeda and Kudo) and wall effects in the counter.[15,25] Some prior work has found it useful to fit the full-energy neutron peak as the sum of two independent Gaussians.[11,26]

Further work is planned to investigate the bestachievable energy resolution from the C-S spectrometer using dPSA techniques, and to study the spectral response of the device, once a higherresolution digitizer is available. A frequent application of these instruments is the study of betadelayed neutron emitters following fission.[27] We intend to use the C-S spectrometer to study the betadelayed neutron spectrum from ${ }^{17} \mathrm{~N}$ produced via the ${ }^{18} \mathrm{O}(\gamma, \mathrm{n})$ reaction. We also plan on using the spectrometer as a nuclear forensics diagnostic tool for studying neutron-emitting radionuclide sealed sources. 

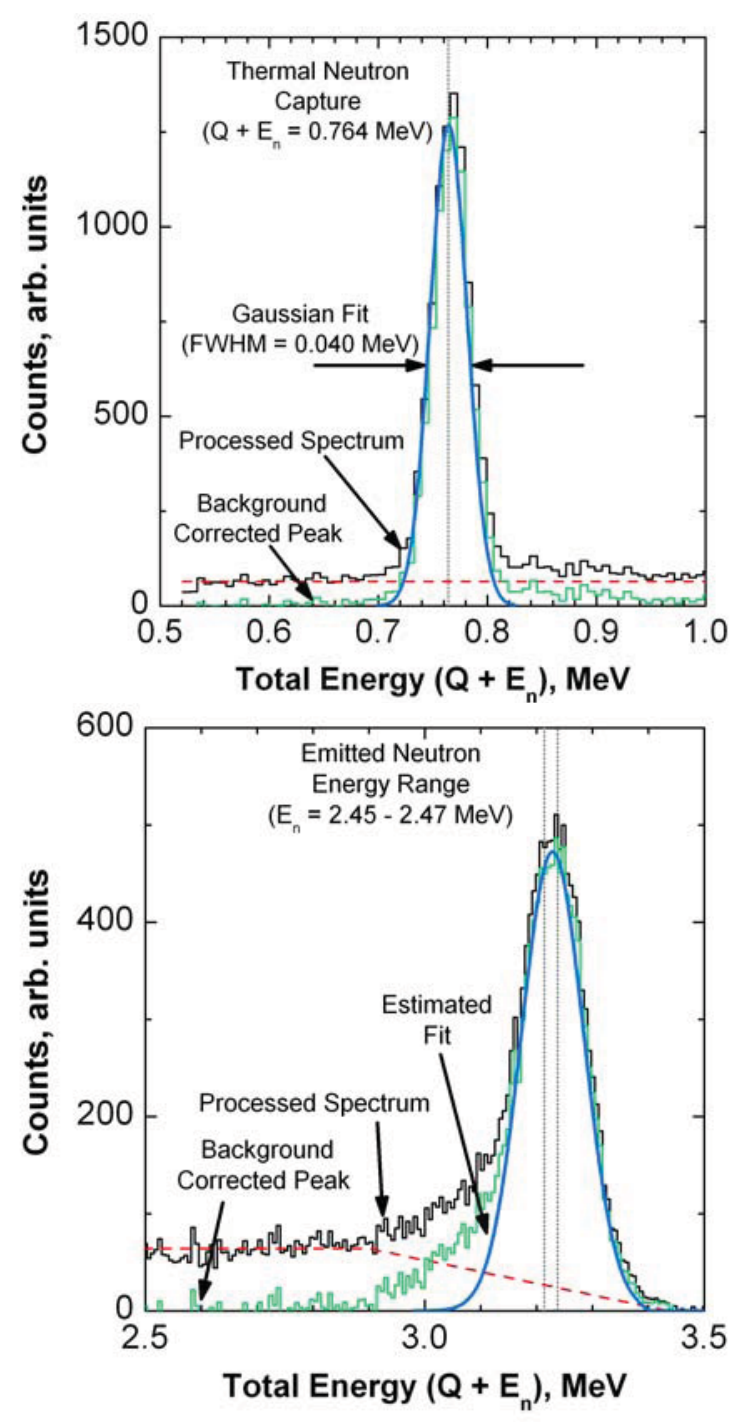

FIGURE 4. The processed DD-ENG neutron spectrum looking at the $\mathrm{E}_{\mathrm{n}}=0 \mathrm{MeV}$ peak (thermal neutrons, top) and the $\mathrm{E}_{\mathrm{n}}=2.46 \mathrm{MeV}$ peak (DD neutrons, bottom). Also shown in these figures is a room-background fit line (red, dashed) and the background-corrected spectrum (green). The dotted line in the top figures represents the Q-value energy for the reaction while the dashed lines in the bottom figure represent the energy region indicated as an angular separation in FIGURE 1, $0.024 \mathrm{keV}$.

\section{ACKNOWLEDGEMENTS}

This work was supported by Idaho National Laboratory as a Laboratory Directed Research and Development funded project. Support was also provided by the U.S. Department of Energy National Nuclear Security Administration Office of Nonproliferation and Verification Research and Development. Idaho National Laboratory is operated for the U.S. Department of Energy by Battelle Energy Alliance under DOE contract DE-AC07-05-ID14517.

\section{REFERENCES}

1. F. D. Brooks and H. Klein, Nucl. Inst. Meth. Phys. Res. A 476 (2002) 1-11.

2. Y. A. Kaschuk, et al., Nucl. Inst. Meth. Phys. Res. A 476 (2002) 511-515.

3. A. Sayres, and M. Coppola, Rev. Sci. Instr. 35 (1964) 431-437.

4. J. M. Cuttler, S. Shalev, and Y. Dagan, Trans. Amer. Nucl. Soc. 12 (1969) 63.

5. J. M. Cuttler, S. Greenberger, and S. Shalev, Nucl. Instr. Meth. 75 (1969) 309-311.

6. W. C. Sailor, and S. G. Prussin, Nucl. Instr. Meth. 173 (1980) 511-515.

7. A. E. Evans, Rad. Effects 96 (1986) 1-4.

8. M. Manolopoulou, et al., Nucl. Inst. Meth. Phys. Res. A 618 (2010) 284-293.

9. H. Ohm, K. L. Kratz, and S. G. Prussin, Nucl. Inst. Meth. Phys. Res. A 256 (1987) 76-90.

10. J. G. Owen, D. R. Weaver, and J. Walker, Nucl. Inst. Meth. 188 (1981) 579-593.

11. W. C. Sailor, S. G. Prussin, and M. S. Derzon, Nucl. Inst Meth. Phys. Res. A 270 (1988) 527-536.

12. A. E. Evans, and J. D. Brandenberger, High Resolution Fast Neutron Spectrometry Without Time-of-Flight, Report LA-UR-78-2562, Los Alamos Scientific Laboratory, Los Alamos, N. M. (1978).

13. W. A. Fisher, et al., Nucl. Inst. Meth. Phys. Res. 219 (1984) 179-191.

14. E. Dietz, et al., Nucl. Instr. Meth. A 332 (1993) 521-528.

15. N. Takeda and K. Kudo, IEEE Trans. Nucl. Sci. 41 (1994) 880-883.

16. H. H. Takahashi, et al., Nucl. Inst. Meth. Phys. Res. A 353 (1994) 164-167.

17. J. Csikai, Handbook of Fast Neutron Generators, Vol. 1, CRC Press Inc., Boca Raton, Fla. (1987).

18. H. Liskien and A. Paulsen, Nucl. Data Tables 11 (1973) 569-619.

19. J. D. Seagrave, et al., $D(d, n) H e^{3}$ and $T(d, n) H e^{4}$ Neutron Source Handbook, Report LAMS-2162, Los Alamos Scientific Laboratory, Low Alamos, N. M. (1958).

20. D. L. Chichester, et al., App. Rad. Iso. 67 (2009) 10131022.

21. D. L. Chichester, J. D. Simpson, and M. Lemchak, J. Radioanal. Nucl. Chem. 271 (2007) 629-637.

22. A. E. Evans, Trans. Am. Nucl. Soc. 38 (1981) 582.

23. A. E. Evans and L. V. East, "Evaluation of a Gridded ${ }^{3} \mathrm{He}$ Spectrometer Tube for Safeguards and Other Nuclear Analysis Applications," Nuclear Analysis Research and Development Program Status Report January-April 1973, Report LA-5291-PR, Los Alamos Scientific Laboratory, Los Alamos, N.M. (1973) 17-19

24. D. L. Chichester, B. W. Blackburn, and A. J. Caffrey, IEEE Trans. Nucl. Sci. 55 (2008) 614-619.

25. S. Shalev, Z. Fishelson, and J. M. Cuttler, Nucl. Inst. Meth. 71 (1969) 292-296.

26. R. Batchelor, R. Aves, and T. H. R. Skyrme, Rev. Sci. Inst. 26 (1955) 1037-1048.

27. H. Franz, et al., Nucl. Inst. Meth. 144 (1977) 253-261. 